\title{
ANÁLISE DAS PUBLICAÇÕES CIENTÍFICAS SOBRE CAPITAL SOCIAL EMPREENDEDOR NO PERÍODO DE 2004 A 2013: UM CAMPO EM EMERGÊNCIA
}

\section{ANALYSIS OF SCIENTIFIC PUBLICATIONS ON ENTREPRENEUR SOCIAL CAPITAL FROM 2004 TO 2013: AN EMERGENT FIELD OF STUDY}

Thiago Roozevelt de Souza

Programa de Mestrado em Administração - Universidade Federal de Sergipe

São Cristóvão, SE, Brasil

E-mail: trsouza@infraero.gov.br

Rivanda Meira Teixeira *

Programa de Mestrado em Administração - Universidade Federal de Sergipe

São Cristóvão, SE, Brasil

E-mail: rivandateixeira@gmail.com

\section{RESUMO}

O estudo do capital social empreendedor é um enfoque emergente nas pesquisas em empreendedorismo. Visa compreender a criação de empresas como um resultado das interações sociais e dos relacionamentos especialmente com indivíduos, instituições e comunidades. Esse estudo procura analisar a produção científica internacional e nacional sobre capital social empreendedor no período de dez anos (2004-2013). O levantamento das publicações foi realizado no Portal de Periódicos da Coordenação de Aperfeiçoamento de Pessoal de Nível Superior (CAPES) e na Scientific Periodicals Electronic Library (SPELL). Essa pesquisa se caracteriza como quantitativa, de natureza descritiva e a amostra foi composta de cinquenta e nove publicações. Foi evidenciado que o periódico com maior número de publicações é o "Entrepreneurship Theory and Practice"; que R. S. Burt é o autor mais referenciado nos artigos internacionais, enquanto $\mathrm{H}$. E. Aldrich é o mais referenciado nos artigos nacionais. Observou-se ainda um crescimento exponencial das publicações nessa temática.

Palavras-chave: Empreendedorismo. Capital social. Capital social empreendedor. Produção científica. estudo bibliométrico.

\section{ABSTRACT}

The study on entrepreneurial social capital is an emerging approach on entrepreneurship field. Aims to understand business creation because social interactions and relationships especially with individuals, institutions and communities. This study analyzes the international and national scientific production about entrepreneur social capital in a period of ten years (2004-2013). Searches were conducted in publications from the Portal from the Coordination of Improvement of Higher Education (CAPES) and in the Scientific Periodicals Electronic Library (SPELL). This research is characterized as quantitative, descriptive in nature and the sample consisted of fifty-nine publications. It was shown that the journal with the highest number of publications is the "Entrepreneurship Theory and Practice"; that R. S. Burt is the most referenced author in international articles while H. E. Aldrich is the most referenced in national articles. It was also observed an exponential growth of publications on this topic.

Keywords: Social capital. Entrepreneur's social capital. Entrepreneurship. scientific production. Bibliometric study.

Data de submissão: 24 de dezembro de 2014.

Data de aprovação: 22 de julho de 2015. 


\section{INTRODUÇÃO}

A atividade empreendedora busca obter vantagem competitiva como forma de lograr êxito, e essa vantagem não é determinada unicamente pelo capital físico, mas é essencialmente ditada pelos ativos estratégicos, como o conhecimento, a propriedade intelectual e as redes sociais do empreendedor, que geram sucesso organizacional ao longo prazo (MIHAELA-RALUCA, 2013).

Granovetter (1973) e Coleman (1988) foram alguns dos pioneiros nos estudos de capital social, originários inicialmente da Sociologia. O estudo do capital social começou a partir da evolução das pesquisas sobre empreendedorismo, que inicialmente eram focadas no indivíduo, mas que a partir dos anos 1980 abrangeu a importância dos contatos sociais, relacionamentos e conexões entre eles (COPE; JACK; ROSE, 2007). Mas foi com o estudo seminal de Adler e Kwon (2002) que de fato se introduziu o conceito de capital social nos estudos organizacionais, ao tomar-se um conceito "guarda-chuva" (algo que abrange uma gama de diferentes significados) e estabelecer, com base nas pesquisas sobre capital social até então realizadas, perspectivas para as pesquisas vindouras - dentre elas o foco no capital social empreendedor.

Considerando que outros aspectos do capital social já foram pesquisados, a abordagem que relaciona capital social e a criação de novas empresas merece um estudo aprofundado, ainda mais por ser recente na pesquisa acadêmica, não sendo identificado até o momento revisão bibliográfica sobre o tema. Uma forma adequada de conhecer o presente estado da arte é através de pesquisa bibliométrica, técnica quantitativa e estatística de medição dos índices de produção e disseminação do conhecimento científico, de forma a efetuar uma avaliação objetiva da produção científica (ARAÚJO, 2006).

Assim, o presente estudo procura analisar a produção científica nacional e internacional, num período de dez anos (2004-2013), a partir de buscas realizadas no Portal de Periódicos da Coordenação de Aperfeiçoamento de Pessoal de Nível Superior (CAPES) e na biblioteca eletrônica Scientific Periodicals Electronic Library (SPELL ${ }^{\circledR}$ ). Especificamente buscou-se apontar os periódicos com maior número de publicações, identificar os autores mais referenciados, descobrir quais são as palavras-chave mais citadas, verificar os enfoques metodológicos mais utilizados e, por fim, identificar a evolução anual das publicações. A seguir será apresentada revisão sobre capital social empreendedor, tema central desse estudo.

\section{CAPITAL SOCIAL EMPREENDEDOR}

Mais do que características psicológicas de um indivíduo, o empreendedorismo é acima de tudo a criação de empresas através de um indivíduo específico, chamado empreendedor (GARTNER, 1989). E para que a criação do novo negócio seja bem-sucedida, um dos fatores de sucesso, que permite ao empreendedor mobilizar relações e recursos que de outra forma seriam mais difíceis de obter, é o seu capital social (BORGES; FILION, 2012).

Não existe um consenso referente ao real significado da expressão "capital social", cujo conceito possui raízes na sociologia. Coleman (1988) aponta que o capital social é definido por sua função, sendo um recurso para as pessoas e produzido a partir de certas relações que geram determinadas ações, dependendo da ação individual para a produção de um bem coletivo e é sustentado por dois pilares, a confiança e a reciprocidade. 
A definição de Nahapiet e Ghoshal (1998) para o capital social é a da soma dos recursos reais e potenciais inerentes a uma rede de relacionamentos de um indivíduo ou unidade social (uma organização, por exemplo), interligando três dimensões: estrutural (configuração da rede de relacionamentos), relacional (a confiança, as normas, as obrigações, a identificação com o grupo) e cognitiva (representações e simbolismos partilhados).

A dimensão estrutural reflete um aspecto "físico" do capital social (capital financeiro, espaço, instalações, equipamento e mão-de-obra). Em se tratando do levantamento de recursos e como a estrutura influencia essa questão, Zhao, Ritchie e Echtner (2011) citam como exemplo os bancos comerciais, que na maioria dos casos dificultam a concessão de empréstimos pela ausência de garantias, o que leva a uma legião de pequenos empreendedores a buscar capital a partir de familiares ou de recursos próprios, o que limita o tamanho e a expansão do negócio, demonstrando que a estrutura do capital social influencia o desenvolvimento de um novo negócio.

Um dos fatores que compõem o capital social (às vezes se confundindo com ele próprio) é o estudo dos relacionamentos/redes sociais, que está ligado à dimensão relacional. Nesse campo, Granovetter (1973) introduziu o conceito de laços, ao analisar as interações entre indivíduos a partir da sua "força" (combinação de fatores como o tempo gasto, intensidade emocional, intimidade e serviços recíprocos de uma relação). Os laços fortes são as relações que fazem parte do cotidiano de um indivíduo e cuja interação é frequente, geralmente da família. Já os laços fracos, caracterizados pela menor intensidade e frequência, nem por isso são menos importantes - pelo contrário, são indispensáveis para a busca das oportunidades por indivíduos e na sua integração na comunidade.

Para Granovetter, saber qual a relação entre a força e o grau de especialização de laços, ou entre a força e estrutura hierárquica, bem como descobrir qual é a sequência de desenvolvimento da estrutura de rede ao longo do tempo são questões importantes para a pesquisa na área de relacionamentos.

Em seu estudo sobre redes sociais, Birley (1985) segue uma linha semelhante, porém com uma terminologia diferente, ao compreender que os empreendedores, durante a abertura de um novo negócio, recebem o auxílio de redes formais (bancos, contadores) e informais (família, amigos); dessa forma, o capital social permite o acesso a recursos tangíveis, tais como capital físico e financeiro, assim como a recursos intangíveis (reputação e conhecimento tácito) - ambos influenciam substancialmente a constituição da empresa.

A dimensão cognitiva exerce influência sobre o empreendedorismo a partir de dois mecanismos, conforme apontam Liao e Welsch (2005): a legitimidade organizacional e a cultura empreendedora. O primeiro mecanismo trata da aceitação do negócio da empresa por parte da sociedade, o que caso não ocorra, traz dificuldades para a sobrevivência da empresa, principalmente em termos de obtenção de informações e recursos. Já o segundo indica que uma comunidade onde a cultura empreendedora é mais forte, as pessoas são mais propensas a tolerar comportamentos de risco e o fracasso, incentivar a independência financeira dos jovens e o auto emprego, proporcionando um ambiente favorável para o acesso à informações e recursos para a criação de novas empresas.

Para o empreendedorismo, o capital social é um fator-chave, tendo influência positiva sobre a inovação, nos custos de transação entre os indivíduos e entidades, custos de negociação, informações e custo do conhecimento e de tomada de decisões (MIHAELA-RALUCA, 2013). Os benefícios do capital social 
também podem ser vistos numa dimensão de comunidade, a partir da confiança social, da participação em organizações voluntárias e da heterogeneidade no acesso ao capital social comunitário e nas atividades empreendedoras (KWON; HEFLIN; RUEF, 2013). Ele também facilita a adoção de inovações internas e externas ao mercado, o que faz parte do conceito de inovação aberta, sendo esta influenciada em seu desempenho pelo capital social (RASS et al., 2013).

Só é possível gerar capital social a partir de interações entre indivíduos e sociedades (BAKER; ONYX; EDWARDS, 2011). Ele proporciona ao empreendedor, dentre outras coisas, acesso a informações e ideias com potencial de serem oportunidades de negócio, o que é potencializado pela diversidade da rede de contatos do empreendedor, além de um maior número de contatos fracos e de buracos estruturais (ausência de ligação entre dois contatos de uma rede de relações), bem como outros tipos de contato podem garantir recursos financeiros, acesso à informações e conhecimentos tecnológicos, legitimação do empreendedor e da empresa no mercado, fonte de sócios e parceiros para o empreendedor e produtividade nas relações com esses sócios e parceiros (BORGES, 2011).

Em comunidades com acesso difícil a crédito, o capital social pode funcionar até como um substituto aos meios formais de obtenção de recursos (BAUERNSCHUSTER; FALCK; HEBLICH, 2010). Da mesma forma, o estudo de Kwon e Arenius (2010) aponta o capital social como um atributo de uma nação, onde a confiança generalizada e associações organizacionais forma um círculo virtuoso. Se junta a isso o entendimento de que as oportunidades empresariais surgem do acesso diferenciado dos indivíduos à informação, influenciado pela confiança generalizada e da importância dos laços fracos (organizações formais de suporte) para acesso ao capital financeiro. Na linha de estudos sobre etnia, Perreault et al. (2007) cita que o capital social é refletido a partir do número de horas dedicadas ao trabalho em rede ou na adesão voluntária em organizações comerciais, sendo que o fator étnico influencia diversas decisões e ações tomadas por tais empreendedores.

Para Nahapiet e Ghoshal (1998), em termos organizacionais, um dos benefícios do capital social é a redução dos custos de transação, ao tornar mais fácil a interação entre agentes econômicos a partir do fluxo livre da informação. Já Adler e Kwon (2002) apontam o goodwill (termo cuja tradução para o português se aproxima da ideia de valor gerado a partir de elementos não-materiais) como a essência do capital social, cujo efeito é percebido a partir do fluxo de informação, influência e solidariedade. Os autores também estabelecem que a estrutura social é categorizada a partir das dimensões da estrutura social, podendo estar enraizadas nas relações de mercado, relações hierárquicas e relações sociais - sendo esta última a dimensão da estrutura social em que o capital social se fundamenta.

Para Sen e Cowley (2013), a sobrevivência é um grande desafio para as pequenas empresas, e uma das formas para os seus proprietários-gestores de aumentar o capital social para superar esse desafio e compensar a sua limitada capacidade de recursos é a partir da Responsabilidade Social Corporativa (RSC). O aumento do capital social da pequena empresa, através da sua participação em ações de RSC podem trazer benefícios à sociedade, fazendo a ponte entre os objetivos de negócios internos e as aspirações sociais.

O capital social, entretanto, não traz somente benefícios. Para Westlund e Bolton (2003), o capital social pode ser contra produtivo ao ponto de restringir a iniciativa empresarial, afirmação essa corroborada por Adler e Kwon (2002), ao estabelecer que o capital social de grupos poderosos pode obstruir a criação de 
negócios por parte de grupos menos favorecidos, tendo como exemplo algum grupo que possui fortes laços internos advindos de normas ou crenças, mas que adotem uma postura xenófoba para com indivíduos que residam na localidade e que estejam fora do grupo.

A compreensão das vantagens e desvantagens do capital social deve ser feita de modo cuidadoso, de forma a melhor entender a importância do capital social para o empreendedorismo. Light e Dana (2013) demonstram que a literatura erra nos argumentos a favor e contra o capital social empreendedor. Quanto às vantagens, o estudo demonstra o equívoco de se crer que o empreendedorismo emerge principalmente do capital social (desconsiderando fatores culturais), a partir de exemplos de comunidades com um capital social abundante, mas que não redundam em novas empresas. Por outro lado, também é errada a afirmação de que o empreendedorismo de grupos menos poderosos é barrado pelo capital social de grupos poderosos, o que o estudo demonstra não ser verdade em todos os casos.

Diversos estudos buscaram compreender o fenômeno empreendedor a partir de modelos teóricos ou modelos econométricos, considerando diversas variáveis. Dinda (2008) apresentou um modelo cujas variáveis eram os capitais social e humano, a produção e o bem-estar, desejando assim expressar o desenvolvimento do capital social por meio da formação de capital humano, criado a partir do consumo produtivo. Já Rass et al. (2013) propuseram um modelo que conceitua o capital social como um mediador entre a implementação das ferramentas de inovação aberta e desempenho da empresa, através de quatro categorias de inovação aberta: por divulgação, por venda, por terceirização e por aquisição - em todas essas categorias o capital social possui um papel específico ao longo do processo, reduzindo a assimetria de informações entre as partes.

Valendo-se de novos conceitos, Westlund e Bolton (2003) mostram que vários aspectos do empreendedorismo podem facilitar ou inibir aspectos do capital social, e eles estabelecem o conceito de "empreendedorismo coletivo", com base no fato de certas regiões terem uma personalidade específica como resultado combinado da personalidade do indivíduo, das interações sociais e empresariais, das redes, instituições, estruturas de mercado e também de fatores fortuitos.

As pesquisas empíricas já realizadas ao longo do tempo apresentam as várias facetas do impacto do capital social na economia. Kim e Kang (2014) apontam que a confiança em estranhos ou em instituições públicas e a ênfase dos pais nas realizações individuais em relação às relações interpessoais no aumento da sua criança estão positivamente associadas com o empreendedorismo. Já Davidsson e Honig (2003), com base em variáveis dependentes relacionadas ao descobrimento e exploração da oportunidade, bem como variáveis específicas para o capital humano e o capital social, descobriram que apenas um aspecto do capital social (ser membro de uma rede de negócios) tendo um efeito positivo estatisticamente significativo, e que o valor de alguns programas de assistência a empreendedores nascentes é questionado.

Baker, Onyx e Edwards (2011) apontaram, a partir de uma pesquisa exploratória, que a interação positiva entre os aspectos relacionais do capital social (manutenção da confiança, incentivo da diversidade e a reciprocidade dentro da formação das empresas e de redes de empresa) é necessária para o êxito de uma empresa. De forma semelhante, Kwon e Arenius (2010) se basearam em dados secundários para estabelecer que o capital social em nível nacional também gera efeitos significativos para a percepção da oportunidade, e que altos níveis de confiança da sociedade podem compensar a falta de capital social em nível individual. 
O estudo do capital social em pesquisas futuras deve seguir algumas diretrizes. Conforme explicam Gedajlovic et al. (2013), pesquisas devem considerar os seguintes fatores: antecedentes e relacionamentos/redes (também diferenciando estes do capital social propriamente dito), resultados do capital social, considerações em múltiplos níveis, as influências do contexto (podendo ser organizacional, institucional ou temporal), desvantagens do capital social, modelos em múltiplos níveis e as dimensões do contexto, mostrando a ampla possibilidade de crescimento nas pesquisas sobre a temática.

Da mesma forma, Cope, Jack e Rose (2007) sugerem áreas importantes para futuras pesquisas, como: a influência das redes sociais na forma de agir dos empreendedores, a complexidade do capital social para diferentes grupos étnicos, as diferenças e semelhanças entre o empreendedorismo privado e o social, e vários outros. Pode-se refletir, sobre esse aspecto, que o capital social, ao gerar ganhos que não são contabilizados formalmente, pode levar a uma perspectiva incorreta de esse fator não ser realmente essencial, mas seus resultados podem ser evidenciados de forma indireta, através da mobilização de recursos e contatos que viabilizam todo empreendimento. É assim que podemos considerar o capital social empreendedor como um campo em emergência, dada a sua importância para a atividade empresarial.

\section{METODOLOGIA}

A presente pesquisa classifica-se, quanto à sua natureza, como descritiva, pois descreve os resultados encontrados após identificar a produção acadêmica sobre capital social empreendedor. Já quanto à estratégia para a coleta dos dados, optou-se pela técnica da bibliometria, que através do uso de métodos quantitativos para uma avaliação objetiva da produção científica, efetua uma leitura mais rica da realidade (ARAÚJO, 2006).

A abordagem utilizada pela presente pesquisa é a quantitativa, pois sua coleta resulta em dados numéricos e padronizados e a análise é conduzida através do uso de diagramas e análise estatística (SAUNDERS; LEWIS; THORNHILL, 2007). Seu horizonte temporal é longitudinal e considera um corte temporal de dez anos, de 2004 até 2013. Também se trata de uma pesquisa documental, em que as informações necessárias para a realização do estudo vieram de documentos já publicados em periódicos científicos.

As fontes de coleta de dados utilizadas foram o Portal de Periódicos da Coordenação de Aperfeiçoamento de Pessoal de Nível Superior (CAPES), para as publicações internacionais e na biblioteca eletrônica Scientific Periodicals Electronic Library (SPELL ${ }^{\circledR}$ ), para as nacionais. A escolha das referidas bases de dados se deu pelos critérios de acessibilidade e facilidade na busca dos artigos. Após a escolha dos meios de coleta de dados, iniciou-se os procedimentos de busca e análise dos artigos. As palavras-chave utilizadas na pesquisa das publicações internacionais foram "social capital" e "entrepreneurship"; já na nacional, as palavras-chave foram "capital social" e "empreendedorismo". Na pesquisa realizada no mês de julho de 2014, foram localizados 4.217 artigos nos periódicos Capes e 31 no SPELL ${ }^{\circledR}$.

Como forma de filtrar os resultados obtidos na pesquisa feita nos Periódicos Capes, foram seguidos os seguintes passos: filtro dos resultados selecionando os periódicos revisados por pares, seleção dos artigos da base de dados Scopus e, por fim, análise dos artigos que tratam sobre capital social e empreendedorismo (ambas as expressões necessitam aparecer no título e/ou no resumo). O número de artigos foi reduzido para 227. 
Em seguida, foi efetuada uma análise dos resumos dos artigos como forma de verificar se os artigos estão relacionados à temática de capital social empreendedor. Assim, foram selecionados cinquenta e cinco artigos internacionais e 9 artigos nacionais. Logo depois, após leitura criteriosa dos artigos selecionados e excluindo possíveis artigos repetidos, gerou-se uma amostra de cinquenta e um artigos internacionais e oito artigos nacionais, totalizando cinquenta e nove artigos, de forma a atingir o objetivo da pesquisa bibliométrica em mensurar o conhecimento ou a compreensão de um dado campo científico (TAGUE-SUTCLIFFE, 1992).

\section{RESULTADOS}

Os resultados encontrados na pesquisa são apresentados para cada objetivo especifico: periódicos com maior número de publicações, autores mais referenciados, as palavras-chave mais frequentes, a evolução anual do número de publicações e os enfoques metodológicos mais utilizados.

\section{Periódicos com maior número de publicações sobre capital social empreendedor}

Foram encontrados 28 diferentes periódicos internacionais, com 51 artigos. A Tabela 1 apresenta os nomes dos periódicos com maior número de publicações, o seu fator de impacto e a quantidade de artigos.

Tabela 1 - Periódicos internacionais por fator de impacto e número de artigos

\begin{tabular}{|c|c|c|}
\hline Nome do periódico & $\begin{array}{l}\text { Fator de } \\
\text { impacto }\end{array}$ & Quantidade \\
\hline Entrepreneurship Theory and Practice & 2,447 & 8 \\
\hline International Small Business Journal & 1,397 & 6 \\
\hline Journal of Business Venturing & 3,265 & 5 \\
\hline Journal of Enterprising Communities: People and Places in the Global Economy & - & 3 \\
\hline International Entrepreneurship and Management Journal & - & \multirow{5}{*}{2} \\
\hline International Journal of Entrepreneurial Behaviour \& Research & - & \\
\hline Journal of Small Business Management & 1,361 & \\
\hline Research Policy & 2,598 & \\
\hline Small Business Economics & 1,641 & \\
\hline Gender in Management: An International Journal & - & \multirow{19}{*}{1} \\
\hline Industrial Management \& Data Systems & - & \\
\hline Journal of Business Research & 1,306 & \\
\hline Journal of Economic Behavior \& Organization & - & \\
\hline Journal of Economic Psychology & - & \\
\hline Journal of International Development & - & \\
\hline Journal of Organizational Change Management & 0,744 & \\
\hline Journal of Small Business and Enterprise Development & - & \\
\hline Journal of Vocational Behavior & 2,033 & \\
\hline Management Decision & - & \\
\hline Management Research News & - & \\
\hline Management Research Review & - & \\
\hline Management Science & - & \\
\hline R\&D Management & - & \\
\hline Regional Science and Urban Economics & - & \\
\hline The International Entrepreneurship and Management Journal & - & \\
\hline The Journal of Socio-Economics & - & \\
\hline Thunderbird International Business Review & - & \\
\hline Women in Management Review & - & \\
\hline TOTAL & & 51 \\
\hline
\end{tabular}

Fonte: Pesquisa nos Periódicos Capes, julho 2014 
No caso dos periódicos nacionais, como demonstra a Tabela 2, são poucos os que se destacam, em função da pequena quantidade de artigos, oito, no total. Seis periódicos diferentes foram listados e os que se destacam, com dois artigos cada, são os periódicos "Cadernos EBAPE.BR" e a "Revista de Administração de Empresas".

Tabela 2 - Periódicos nacionais pela quantidade de artigos

\begin{tabular}{l|c}
\hline \multicolumn{1}{c|}{ Nome do periódico } & Quantidade \\
\hline Cadernos EBAPE.BR & 2 \\
\cline { 1 - 1 } Revista de Administração de Empresas & \\
\cline { 1 - 1 } Revista de Administração e Inovação & \multirow{2}{*}{1} \\
\cline { 1 - 1 } Revista de Empreendedorismo e Gestão de Pequenas Empresas & \\
\hline Revista de Negócios & 8 \\
\hline Turismo em Análise & \\
\hline TOTAL &
\end{tabular}

Fonte: Base Spell, julho 2014.

\section{Autores mais referenciados}

Foi elaborada a Tabela 3, contendo um ranking com dez autores mais referenciados com publicações sobre capital social empreendedor em periódicos internacionais, adaptando a estrutura de análise de Venâncio, Andrade e Fiates (2014). Foram identificados os dez autores mais referenciados no universo de 51 artigos internacionais, com destaque para R. S. Burt, R. D. Putnam, J. S. Coleman e H. E Aldrich.

Tabela 3 - Autores mais referenciados (periódicos internaciona
\begin{tabular}{c|c|c}
\hline Classificação & Autor & Total de Citações \\
\hline $1^{\circ}$ & Burt, R. S. & 58 \\
\hline $2^{\circ}$ & Putnam, R. D. & 48 \\
\hline $3^{\circ}$ & Coleman, J. S. & 46 \\
\hline $4^{\circ}$ & Aldrich, H. E. & 40 \\
\hline $5^{\circ}$ & Bourdieu, P. & 33 \\
\hline \multirow{2}{*}{$6^{\circ}$} & Granovetter, M. & 32 \\
\cline { 2 - 3 } & Shane, S. & \\
\hline $8^{\circ}$ & Nahapiet, J. & 25 \\
\hline $9^{\circ}$ & Adler, P. S. & 22 \\
\hline $10^{\circ}$ & Davidsson, P. & 21 \\
\hline
\end{tabular}

Fonte: Pesquisa nos Periódicos Capes, julho 2014

Já nos periódicos nacionais, foram identificados nos oito artigos que fizeram parte desse estudo, os dez autores com maior número de citações, com destaque para H. E Aldrich, R. S. Burt, R. D. Putnam e M. Granovetter (Tabela 4). 
Tabela 4 - Autores mais referenciados (periódicos nacionais)

\begin{tabular}{c|c|c}
\hline Classificação & Autor & Total de Citações \\
\hline $1^{\circ}$ & Aldrich, H. E. & 25 \\
\hline $2^{\circ}$ & Burt, R. S. & 17 \\
\hline $3^{\circ}$ & Putnam, R.D. & 13 \\
\hline \multirow{2}{*}{$4^{\circ}$} & Granovetter, M. & \multirow{2}{*}{} \\
\cline { 2 - 2 } & Lin, N. & 12 \\
\cline { 2 - 3 } & Johannisson, B. & \\
\hline $7^{\circ}$ & Coleman, J. S. & 11 \\
\hline $8^{\circ}$ & Bourdieu, P. & 10 \\
\hline $9^{\circ}$ & Birley, S. & 9 \\
\hline $10^{\circ}$ & Borges, C. & 8 \\
\hline
\end{tabular}

Fonte: Base Spell, julho 2014.

São comuns às duas listas dos periódicos nacionais e internacionais, os autores R. S. Burt, R. Putnam, H. E. Aldrich, M. Granovetter, J. S. Coleman e P. Bourdieu. Destaca-se também que há apenas um autor brasileiro na lista de autores mais referenciados, C. Borges.

\section{Palavras-chave mais frequentes}

Baseado na estrutura de análise utilizada por Santos, Uriona-Maldonado e Santos (2011), foi elaborada a Tabela 5, que apresenta as dez palavras-chave mais frequentes nos artigos, diferentes das utilizadas nas buscas dos artigos- "capital social" e "empreendedorismo".

Tabela 5 - Palavras-chave mais frequentes

\begin{tabular}{|c|c|}
\hline Palavras & Frequência \\
\hline Relacionamento & 19 \\
\hline Desempenho & 16 \\
\hline Capital & 15 \\
\hline Tecnologia & 13 \\
\hline Organizações & 13 \\
\hline Pequena empresa & 11 \\
\hline Comunidade & 10 \\
\hline Inovação & 08 \\
\hline Sucesso & 08 \\
\hline Minorias & 07 \\
\hline
\end{tabular}

Fonte: Periódicos Capes e Spell, julho de 2014 
A palavra-chave "Relacionamento" abarca estudos com foco específico em aspectos como laços, relacionamentos para abertura do negócio, confiança dos relacionamentos e outros. A palavra "Desempenho" abrange estudos que correlacionam capital social e desempenho organizacional. "Capital" designa os estudos que buscam compreender a importância do capital econômico e de recursos financeiros para a atividade empreendedora, enquanto "Tecnologia" abarca os estudos com foco em questões como internet e sistemas operacionais. A palavra-chave "Organizações" descrevem estudos que tratam de diferentes configurações organizacionais, "Pequena empresa", que trata especificamente deste tipo de configuração organizacional. "Comunidade" reúne publicações a respeito de temas sobre diferentes comunidades e sua influência no capital social empreendedor. "Sucesso" abrange as pesquisas sobre questões de êxito em empreendimentos e sua relação ao capital social, e por fim, "Minorias" reúne os estudos com focos em questões de gênero ou étnicas.

\section{Número de publicações por ano}

O presente estudo adotou um horizonte temporal de dez anos (2004-2013). A Tabela 6 apresenta a distribuição dos artigos sobre capital social empreendedor nesses anos, nos periódicos nacionais e internacionais. Observa-se que nos anos de 2011 e 2013 foram os de maior número de publicações.

Tabela 6 - Distribuição anual de artigos sobre capital social empreendedor (2004-2013) em periódicos nacionais e internacionais

\begin{tabular}{l|c|c|c|c|c|c|c|c|c|c}
\hline Origem do Periódico & $\mathbf{2 0 0 4}$ & $\mathbf{2 0 0 5}$ & $\mathbf{2 0 0 6}$ & $\mathbf{2 0 0 7}$ & $\mathbf{2 0 0 8}$ & $\mathbf{2 0 0 9}$ & $\mathbf{2 0 1 0}$ & $\mathbf{2 0 1 1}$ & $\mathbf{2 0 1 2}$ & $\mathbf{2 0 1 3}$ \\
\hline Internacional & - & 3 & 2 & 7 & 2 & 7 & 5 & 8 & 6 & 11 \\
\hline Nacional & 1 & 1 & - & - & - & - & 2 & 2 & 2 & - \\
\hline Total: & $\mathbf{1}$ & $\mathbf{4}$ & $\mathbf{2}$ & $\mathbf{7}$ & $\mathbf{2}$ & $\mathbf{7}$ & $\mathbf{7}$ & $\mathbf{1 0}$ & $\mathbf{8}$ & $\mathbf{1 1}$ \\
\hline
\end{tabular}

Fonte: Periódicos Capes e Spell, julho de 2014

Em seguida, a figura 1 apresenta informações gráficas da evolução das publicações nacionais e internacionais nos anos de 2004 a 2013. As informações apresentadas na figura 1 permitem afirmar que o campo de estudos sobre capital social empreendedor está em ascensão, tanto nos periódicos internacionais quanto nos nacionais. 
Figura 1 - Distribuição anual de artigos sobre capital social empreendedor (2004-2013) em periódicos nacionais e internacionais

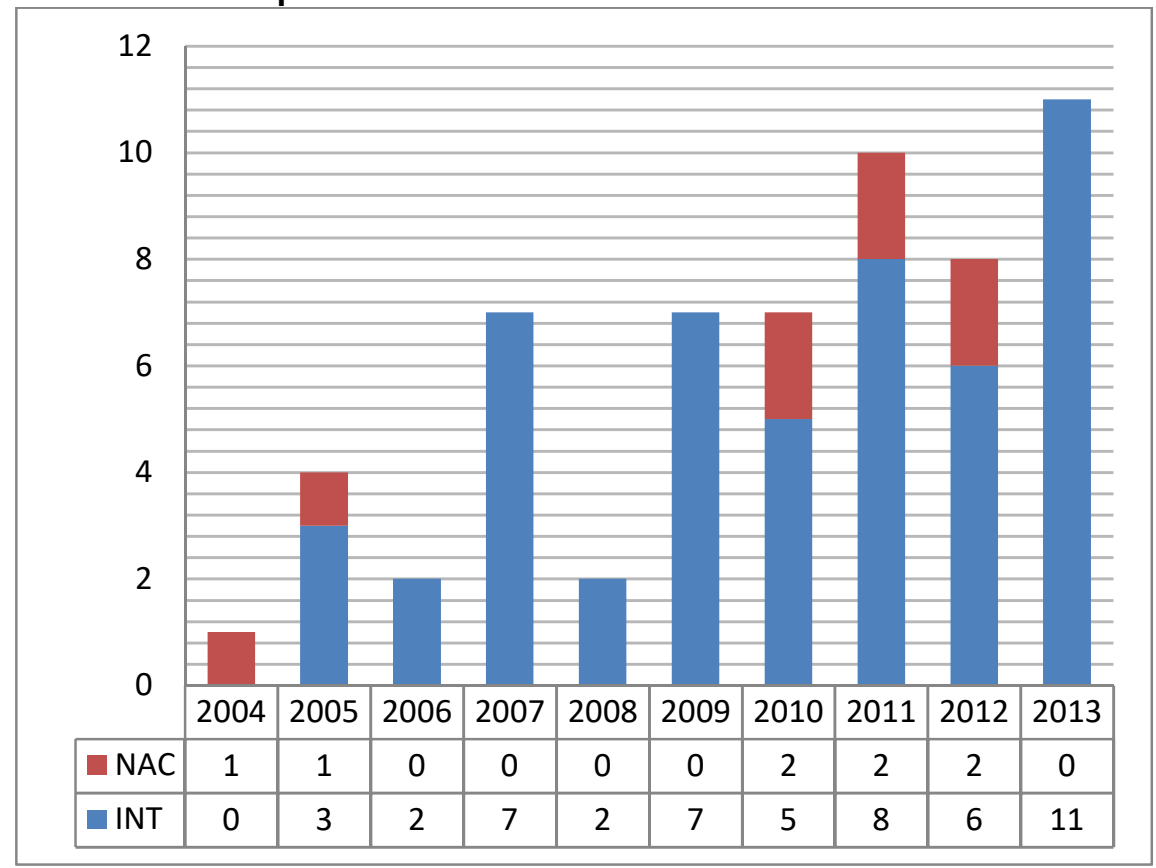

Fonte: Periódicos Capes e Spell, julho de 2014

\section{Enfoques metodológicos adotados nos estudos}

Para o enfoque metodológico dos artigos adotou-se a terminologia de Saunders et al. (2007), que definiu as seguintes estratégias de pesquisa: experimento; survey; estudo de caso; pesquisa-ação; grounded theory; etnografia e pesquisa de arquivo. Para apresentação da estrutura analítica, adotou-se a sugerida por Venâncio, Andrade e Fiates (2014), que apresenta a abordagem, que pode ser qualitativa, quantitativa ou quanti-quali; o objetivo da pesquisa que pode ser exploratório ou descritivo; a estratégia de pesquisa, que pode ser estudo de caso único, estudo de casos múltiplos, survey, pesquisa documental, multi-métodos e outros e as técnicas de coletas de dados, que podem ser entrevista, questionário, dados secundários e outros. Os resultados são apresentados na Tabela 7, a seguir. 
Tabela 7 - Enquadramentos metodológicos utilizados nos artigos nacionais e internacionais

\begin{tabular}{|c|c|c|c|}
\hline \multicolumn{2}{|c|}{ Enquadramento Metodológico } & \multirow{2}{*}{$\begin{array}{c}\text { Art. internacionais } \\
16\end{array}$} & \multirow{2}{*}{$\begin{array}{c}\text { Artigos nacionais } \\
7\end{array}$} \\
\hline \multirow{3}{*}{ Abordagem } & Qualitativa & & \\
\hline & Quantitativa & 33 & 1 \\
\hline & Quanti-quali & 2 & - \\
\hline \multirow{2}{*}{$\begin{array}{c}\text { Objetivos da } \\
\text { pesquisa }\end{array}$} & Exploratória & 5 & 2 \\
\hline & Descritiva & 46 & 6 \\
\hline \multirow{6}{*}{$\begin{array}{c}\text { Estratégia de } \\
\text { pesquisa }\end{array}$} & Estudo de caso único & 1 & - \\
\hline & Estudo de casos múltiplos & 6 & 5 \\
\hline & Survey & 33 & 1 \\
\hline & Documental & 5 & 2 \\
\hline & Multi-métodos & 2 & - \\
\hline & Outros & 4 & - \\
\hline \multirow{4}{*}{$\begin{array}{c}\text { Técnicas de } \\
\text { coletas de dados }\end{array}$} & Entrevista & 10 & 5 \\
\hline & Questionário & 20 & 1 \\
\hline & Dados secundários & 13 & - \\
\hline & Outros & 8 & 2 \\
\hline
\end{tabular}

Fonte: Periódicos Capes e Spell, julho de 2014

Foi evidenciada a preferência das pesquisas nacionais pelas abordagens qualitativas, diferentemente das pesquisas internacionais, em que os métodos quantitativos são mais utilizados, seja a partir da aplicação de questionários ou da análise de dados secundários, com análises estatísticas diversas, a exemplo de análise de regressão, de correlação e de outras. As pesquisas internacionais sobre capital social empreendedor também se caracterizam pelo uso de estratégias pouco usuais no Brasil, como a etnografia e o focus group. Nas pesquisas nacionais, a preferência é pelo método de estudo de casos múltiplos.

\section{CONCLUSÕES}

O capital social é um campo de pesquisa emergente nos estudos sobre empreendedorismo considerando os impactos do capital social na atividade empreendedora. Este estudo analisou a produção científica nacional e internacional sobre capital social empreendedor entre 2004 e 2013, efetuando buscas no Portal de Periódicos da Coordenação de Aperfeiçoamento de Pessoal de Nível Superior (CAPES) e na base eletrônica Scientific Periodicals Electronic Library (SPELL ${ }^{\circledR}$ ). Foram localizados cinquenta e um artigos internacionais e oito nacionais, totalizando cinquenta e nove artigos. Foi evidenciado que o periódico com maior número de publicações sobre capital social empreendedor é o "Entrepreneurship Theory and Practice"; que R. S. Burt é o autor mais referenciado nos artigos internacionais, enquanto H. E. Aldrich é o mais referenciado nos artigos nacionais, destacando-se também os autores R. D. Putnam, J. S. Coleman, P. Bourdieu e M. Granovetter como os mais citados tanto nos periódicos nacionais como nos internacionais. As pesquisas com abordagem quantitativa são mais comuns nos estudos internacionais, enquanto nos nacionais há uma preferência pelas abordagens qualitativas; observou-se que no período de 2004 a 2008 foram 
publicados dezesseis artigos e de 2009 a 2013 foram publicados quarenta e três, mostrando o crescimento exponencial das publicações nessa temática.

Com base nos resultados obtidos, uma agenda de pesquisa pode ser proposta com os seguintes aspectos: 1 - descobrir os artigos sobre capital social empreendedor em diferentes tipologias de periódicos ou congressos; 2 - em relação aos autores que influenciam o campo de pesquisa, é necessário que sejam efetuadas triangulações com outros campos ou perspectivas de pesquisa; 3 - em relação às palavras-chaves, descobrir quais são as tendências, em termos de perspectivas teóricas e metodológicas, dos estudos contemporâneos; 4 - verificar se o crescimento no número de artigos publicados se alinha com um crescimento da qualidade ou relevância; 5 - verificar se o crescimento da qualidade dos estudos se dá por um refinamento no aparato metodológico.

O número reduzido de artigos selecionados pode ser considerado uma limitação do estudo. Futuros levantamentos podem abordar essa temática, utilizando-se de técnicas estatísticas e análises diferenciadas das utilizadas no presente estudo, ou abordagens diferenciadas, especialmente com foco quantitativo, ainda pouco adotada nos estudos nacionais. Isso está no âmago da importância dos estudos bibliométricos para o progresso de um campo científico, conforme o entendimento de Tague-Sutcliffe (1992), que apresenta os benefícios em termos de se estabelecer uma visão da atividade científica de uma dada região ou localidade, realizar comparações longitudinais, de forma a avaliar a importância de uma dada área do conhecimento, não sendo diferente com o capital social empreendedor. No entanto, embora tenha utilizado com uma amostra relativamente pequena de artigos, esse levantamento reflete a produção acadêmica recente nacional e internacional na área de capital social empreendedor que pode servir de subsídio para novas pesquisas na área, visto se tratar de uma temática inovadora e ainda em estágio inicial no Brasil.

\section{REFERÊNCIAS}

ADLER, P. S.; KWON, S. W. Social capital: Prospects for a new concept. Academy of Management Review, v. 27, n. 1, p. 17-40, 2002.

ARAÚJO, C. A. Bibliometria: evolução histórica e questões atuais. Em Questão, v. 12, n. 1, p. 11-32, 2006.

BAKER, E.; ONYX, J.; EDWARDS, M. Emergence, social capital and entrepreneurship: Understanding networks from inside. E:CO Issue, v. 13, n. 3, p. 21-38, 2011.

BAUERNSCHUSTER, S.; FALCK, O.; HEBLICH, S. Social capital access and entrepreneurship. Journal of Economic Behavior \& Organization, v. 76, n. 3, p. 821-833, 2010.

BIRLEY, S. The role of networks in the entrepreneurial process. Journal of Business Venturing, v.1., n.1, p. 107-117, 1985.

BORGES, C. O papel do capital social do empreendedor na criação de empresas tecnológicas. Revista de Administração e Inovação, v. 8, n. 2, p. 162-181, jul./set. 2011.

BORGES, C.; FILION, L. J. Evolução do capital social empreendedor dos spin-offs universitários. REGEPE - Revista de Empreendedorismo e Gestão de Pequenas Empresas, v. 1, n. 1, p. 3-31, 2012.

COLEMAN, J. S. Social capital in the Creation of Human Capital. American Journal of Sociology, v. 94, p. 95-120, 1988.

COPE, J.; JACK, S.; ROSE, M. B. Social Capital and Entrepreneurship: An Introduction. International Small Business Journal, v. 25, n. 3, p. 213-220, 2007.

DAVIDSSON, P. HONIG. B. The role of social and human capital among nascent entrepreneurs. Journal of Business Venturing, n. 18, p. 301-331, 2003.

DINDA, S. Social capital in the creation of human capital and economic growth: A productive consumption approach. The Journal of Socio-Economics, n. 37, p. 2020-2033, 2008.

GARTNER, W. B. "Who is an entrepreneur?" is the wrong question. Entrepreneurship Theory and Pratice, v. 13, p. 4767, 1989. 
GEDAJLOVIC, E.; HONIG, B.; MOORE, C. B.; PAYNE, G. T.; WRIGHT, M. Social capital and Entrepreneurship: A Schema and Research Agenda. Entrepreneurship Theory and Pratice, v. 37, n. 3, p. 455-478, 2013.

GRANOVETTER, M. S. The strength of weak ties. American Journal of Sociology, v. 78, n. 6, 1973.

KIM, B. Y.; KANG, Y. Social capital and entrepreneurial activity: A pseudo-panel approach. Journal of Economic Behavior \& Organization, n. 97, p. 47-60, 2014.

KWON, S. W.; ARENIUS, P. Nations of entrepreneurs: A social capital perspective. Journal of Business Venturing, v. 25, n. 3, p. 315-330, 2010.

KWON, S. W.; HEFLIN, C.; RUEF, M. Community Social Capital and Entrepreneurship. American Sociological Review, v. 78, n. 6, p. 980-1008, 2013.

LIAO, J.; WELSCH, H. Roles of Social Capital in Venture Creation: Key Dimensions and Research Implications. Journal of Small Business Management, v. 43, n. 4, p. 345-362, 2005.

LIGHT, I.; DANA, L. P. Boundaries of Social Capital in Entrepreneurship. Entrepreneurship Theory and Pratice, v. $37, \mathrm{n}$. 3, p. 603-624, 2013.

MIHAELA-RALUCA, B.; Social capital framework and its influence on the entrepreneurial activity. Annals of Faculty of Economics, v. 1, n. 1, p. 581-589, 2013.

NAHAPIET, J.; GHOSHAL, S. Social capital, intellectual capital, and the organizational advantage. Academy of Management Review, v. 23, n. 2, p. 242-266, 1998.

PERREAULT, C.; BRENNER, G. A.; MENZIES, T. V.; FILION, L. J.; RAMANGALAHY, C. Social capital and business performance: Ethnic enterprises in Canada. International Journal of Business and Globalisation, v. 1, n. 2, p. 145-160, 2007.

RASS, M.; DUMBACH, M; DANZINGER, F.; BULLINGER, A. C.; MOESLEIN, K. M. Open innovation and firm performance: The mediating role of social capital. Creativity and Innovation Management, v. 22, n. 2, p. 177-194, 2013.

SANTOS, J. L. S.; URIONA-MALDONADO, M.; SANTOS, R. N. M. Inovação e conhecimento organizacional: um mapeamento bibliométrico das publicações científicas até 2009. Revista Organizações em Contexto, v. 7, n. 13, p. 3158, 2011.

SAUNDERS, M.; LEWIS, P.; THORNHILL, A. Research Methods for Business Students. 4. ed. Harlow: Pearson Education, 2007.

SEN, S.; COWLEY, J. The Relevance of Stakeholder Theory and Social Capital Theory in the Context of CSR in SMEs: An Australian Perspective. Journal of Business Ethics, v. 118, p. 413-427, 2013.

TAGUE-SUTCLIFFE, J. An introduction to informetrics. Information Processing and Management, v. 28, n. 1, p. 1-3, 1992.

VENÂNCIO, D. M.; ANDRADE, D.; FIATES, G. G. S. Inovação em Serviços: Um estudo bibliométrico da produção científica no Portal CAPES até setembro de 2013. Revista Eletrônica de Estratégia \& Negócios, v. 7, n. 1, p. 137-157, 2014.

ZHAO, W.; RITCHIE, J. R. B.; ECHTNER, C. M. Social Capital and Tourism Entrepreneurship. Annals of Tourism Research, v. 38, n. 4, p. 1570-1593, 2011. 